\title{
Ödementstauung und Herzinsuffizienz - wann sind Kompression und Manuelle Lymphdrainage gegenangezeigt?
}

\author{
T. Hirsch \\ Praxis für Innere Medizin und Gefäßkrankheiten, Venen Kompetenz-Zentrum ${ }^{\circledR}$, Halle (Saale)
}

\section{Schlüsselwörter \\ Kompressionstherapie, Entstauungstherapie, Manuelle Lymphdrainage, dekompensierte Herzinsuffizienz, Volumenverlagerung}

\section{Zusammenfassung}

Die dekompensierte Herzinsuffizienz gilt als Kontraindikation für Kompressionstherapie und Manuelle Lymphdrainage. Da die Verordnung der genannten Heil- und Hilfsmittel in der Regel durch Gefäßmediziner, Dermatologen und Hausärzte erfolgt, weniger jedoch durch Kardiologen, soll der Zusammenhang zwischen Kompression und Herzinsuffizienz untersucht und der Stellenwert der Herzinsuffizienz definiert werden. Entsprechend der Leitlinie der European Society of Cardiology ist eine Herzinsuffizienz gekennzeichnet durch Luftnot, periphere Ödeme, Palpitationen und Leistungsverlust. Ursächliche Grunderkrankungen sind KHK, Hypertonie, Herzklappenvitien und postentzündliche Veränderungen. Als dekompensierte Herzinsuffizienz sind die Krankheitsstadien NYHA III und IV anzusehen, die durch das Auftreten der o.g. Symptome bei bereits geringer körperlicher Belastung bzw. in Ruhe gekennzeichnet sind. Die Gegenanzeige für Kompressionstherapie bei dekompensierter Herzinsuffizienz ergibt sich aus der Überlegung, dass das Blutvolumen der Extremitäten herzwärts verlagert

Korrespondenzadresse

Dr. med. Tobias Hirsch

Praxis für Innere Medizin und Gefäßkrankheiten

Venen Kompetenz-Zentrum ${ }^{\circledR}$

Leipziger Straße 5

06108 Halle (Saale)

Tel. +49-345-503303

Fax +49-345-5033 04

E-Mail: info@gefaessmedizin-hirsch.de

www.gefaessmedizin-hirsch.de wird und eine Überlastung im kleinen Kreislauf mit Ausprägung eines Lungenödems bewirken kann. Szintigraphische und airplethysmographische Untersuchungen belegen eine Umverteilung regionaler Blutvolumina unter medizinischen Kompressionsstrümpfen (MKS), welche vom herzgesunden Patienten kompensiert wird. Im Falle einer strukturellen Herzerkrankung ist ein verändertes Verhalten des Myokards und der Regulationsvorgänge belegt. Eine erhöhte Volumenbelastung im rechten Vorhof bewirkt einen lokalen Druckanstieg, verbunden mit nachweislich vermehrter Expression natriuretischer Peptide. Untersuchungen belegen jedoch, dass der Anstieg passager ist und nicht zwingend von klinisch relevanten hämodynamischen Veränderungen begleitet wird. So stellen MKS bei der Herzinsuffizienz in den Stadien NYHA I und II keine Gefährdung dar. Invasive Messungen an Patienten der Stadien NYHA III und IV belegen ebenfalls, dass hämodynamische Veränderungen durch Kompression nach wenigen Minuten kompensiert werden und in der Regel nur eine geringe klinische Beeinträchtigung zur Folge haben. Dennoch hat eine entstauende Therapie von Patienten mit dekompensierter Herzinsuffizienz wegen der prognostischen Bedeutung grundsätzlich unter engmaschiger klinischer Kontrolle zu erfolgen.

Oedema Drainage and Cardiac Insufficiency When is there a Contraindication for Compression and Manual Lymphatic Drainage?

Phlebologie 2018; 47: 115-119

https://doi.org/10.12687/phleb2420-3-2018

Eingereicht: 14. März 2018

Angenommen: 16. April 2018

English version available at: www.thieme.de/phlebo

\section{Keywords}

Compression therapy, decongestive therapy, lymphatic drainage, decompensated heart failure, volume shift

\section{Summary}

Decompensated heart failure is considered a contraindication for compression therapy and lymphatic drainage. Since the treatment is usually prescribed by phlebologists, dermatologists and GPs, and less often by cardiologists, it is important to understand the correlation between compression and heart failure, and to establish the significance of the latter. According to the European Society of Cardiology, heart failure is characterised by a shortness of breath, peripheral oedema, palpitations and reduced cardiac output. Underlying primary diseases include CHD, hypertension, valvular heart disease and post-inflammatory changes. Decompensated heart failure is classified under NYHA stages III and IV in which the symptoms mentioned above appear even at low levels of physical activity or when the body is at rest. The contraindication for using compression therapy with decompensated heart failure is based on the idea that blood volumes in the extremities shift towards the heart causing a volume overload in the pulmonary circulation and possibly resulting in a pulmonary oedema. Scintigraphy and air-plethysmography reveal the displacement of regional blood volumes when medical compression stockings (MCS) are used. This is compensated for in healthy heart patients. Structural cardiac disease has been shown to change the behaviour of myocardial regulation processes. An increased volume in the right atrium produces a local rise in pressure and an increased expression of natriuretic peptides. However, studies have shown that this increase is tem- 
porary and is not accompanied by clinically relevant haemodynamic changes. Thus MCSs pose no threat at NYHA stages I and II. Invasive measurements of patients suffering from heart failure NYHA stages III and IV have also identified that haemodynamic changes caused by compression are compensated for after a few minutes and usually only have minor clinical impact. Nevertheless, drainage therapy on patients with decompensated heart failure should be strictly monitored due to its prognostic implications.

\section{Einleitung}

Die dekompensierte Herzinsuffizienz gilt entsprechend deutschen und internationalen Leitlinien als absolute Kontraindikation für den phlebologischen und lymphologischen Kompressionsverband (S2-Leitlinie 2009-2014), Medizinische Kompressionsstrümpfe (S1-Leitlinie 2006-2011) und Manuelle Lymphdrainage (S2-Leitlinie 2017) (1-3). In der Literatur finden sich vereinzelte Fallberichte über die Auslösung einer kardialen Beeinträchtigung aufgrund einer entstauenden Behandlung $(4,5)$. Die Autoren eines aktuellen Reviews von 20 internationalen Leitlinien und Konsensuspapieren zur Therapie des Ulcus cruris aus den Jahren 2009 bis 2016 kommen zu dem Schluss, dass lediglich ein Lungenödem als absolute Kontraindikation zur Kompressionsbehandlung anzusehen ist, wohingegen diese Aussage für die Herzinsuffizienz im Allgemeinen nicht uneingeschränkt geteilt wird (6).

Da die Verordnung der genannten Heilund Hilfsmittel in der Regel durch Gefäßmediziner, Dermatologen und Hausärzte erfolgt, weniger jedoch durch Kardiologen, soll der Zusammenhang zwischen Herzinsuffizienz und Kompressionstherapie näher untersucht werden.

\section{Chronische und dekompen- sierte Herzinsuffizienz}

Entsprechend der aktuellen Leitlinie der European Society of Cardiology (ESC) von 2016 (7), der sich auch die Deutschen Gesellschaft für Kardiologie (DGK) anschließt, handelt es sich bei der Herzinsuffizienz um ein Syndrom, welches gekennzeichnet ist durch Luftnot, Knöchelödeme und allgemeine Müdigkeit. Diese Symptome werden begleitet von klinischen Zeichen wie gestauten Halsvenen, pulmonalen Rasselgeräuschen oder peripheren Ödemen. Als Ursachen werden kardiale Strukturdefekte bzw. Funktionsstörungen benannt, die zu einer verringerten Auswurfleistung des Herzen und/oder zu erhöhten intrakardialen Drücken in Ruhe oder unter Belastung führen. Eine Herzinsuffizienz kann sowohl Folge einer systolischen, als auch - bei normaler Ejektionsfraktion - einer diastolischen Funktionsstörung des Herzens sein. Auslösende Erkrankungen sind koronare Herzkrankheit, Hypertonie,

\begin{tabular}{|l|l|l|}
\hline $\begin{array}{l}\text { NYHA- } \\
\text { Stadium }\end{array}$ & Klinik & ICD-10-Code \\
\hline I & $\begin{array}{l}\text { Keine Einschränkung bei physischer } \\
\text { Belastung. }\end{array}$ & I50.11 \\
\hline II & $\begin{array}{l}\text { Geringfügige Einschränkungen bei nor- } \\
\text { maler physischer Belastung mit Ermü- } \\
\text { dung, Herzrasen und Kurzatmigkeit; keine } \\
\text { Symptome in Ruhe }\end{array}$ & I50.12 \\
\hline III & $\begin{array}{l}\text { Deutliche Einschränkungen bei geringer } \\
\text { physischer Belastung mit Ermüdung, } \\
\text { Herzrasen und Luftnot; } \\
\text { keine Symptome in Ruhe }\end{array}$ & I50.13 \\
\hline IV & $\begin{array}{l}\text { Jede physische Aktivität führt zu Be- } \\
\text { schwerden. Herzinsuffizienzsymptome in } \\
\text { Ruhe mit Verschlimmerung bei Aktivität. }\end{array}$ & I50.14 \\
\hline
\end{tabular}

Tab. 1

Herzinsuffizienz Schweregradeinteilung der New York Heart Association (nach Dolgin 1994 [8]) mit Diagnose-Codierung
Herzklappenvitien und postentzündliche Veränderungen. Zur Bildgebung wird die transthorakale Echokardiographie empfohlen, bei eingeschränkten Ultraschallbedingungen die kardiale Magnetresonanztomographie. Bei Verdacht auf das Vorliegen einer chronischen ischämischen Herzerkrankung sollte eine Koronarangiographie erfolgen (Empfehlungsgrad IC).

In Abhängigkeit vom Schweregrad der Erkrankung können diagnostische Tests wie Laboruntersuchungen (u.a. natriuretische Peptide) oder eine Rechtsherzkatheter-Untersuchung zur Ermittlung der Druckverhältnisse im kleinen Kreislauf Anwendung finden.

International ist die Einteilung der Herzinsuffizienz in 4 Schweregrade nach dem Schema der New York Heart Association (NYHA) etabliert ( $>$ Tab. 1) (8), welche auch Berücksichtigung findet in der Internationalen Klassifikation der Krankheiten (ICD-10).

Eine terminologische Schwierigkeit besteht darin, dass der Begriff der „dekompensierten Herzinsuffizienz" in der NYHA-Einteilung und den ESC-Leitlinien zwar benutzt, aber nicht explizit definiert wird. Legt man zu Grunde, dass es sich bei der Herzinsuffizienz um eine chronische Erkrankung handelt, so muss als „Dekompensation“ eine akute Verschlechterung der Herzfunktion angesehen werden. In der ESC-Leitlinie werden dafür die Begriffe „fortgeschrittene Herzinsuffizienz" („advanced heart failure”) und „akute Herzinsuffizienz" verwendet. Nach NYHA-Klassifikation finden in diesen Fällen die Stadien III und IV Anwendung. Als dekompensierte Herzinsuffizienz ist demnach ein $\mathrm{Zu}$ stand zu bezeichnen, der mit zunehmender Dyspnoe, Müdigkeit und Palpitationen bereits bei geringer körperlicher Belastung oder sogar in Ruhe einhergeht.

Die ESC-Leitlinie benennt explizit Faktoren, welche eine akute Dekompensation der chronischen Herzinsuffizienz triggern können, wie beispielsweise Infektionen, Stress, Operationen. Es muss festgestellt werden, dass weder Kompressionstherapie, noch Manuelle Lymphdrainage in dieser Auflistung als auslösende Faktoren aufgeführt werden. 


\section{Kompressionstherapie bewirkt eine Verschiebung des Blutvolumens}

Ca. $70-85 \%$ des Blutvolumens befinden sich im venösen bzw. kapillären Schenkel des Kreislaufsystems. Daraus ist zu schlussfolgern, dass Kompressionstherapie an den Extremitäten zu einer Verdrängung des Blutvolumens in die Gefäße des Stammes führt. Die Gegenanzeige für Kompressionstherapie bei dekompensierter Herzinsuffizienz ergibt sich aus der Überlegung, dass das aus der Haut und der Muskulatur der Beine bzw. Arme mobilisierte Blut über die untere bzw. obere Hohlvene zum Herzen gelangt und eine Volumenüberlastung bewirkt. Im schlimmsten Falle ist als Ergebnis der Verlagerung peripherer Ödeme in die Gefäße des Stammes aufgrund der Stauung im kleinen Kreislauf die konsekutive Ausprägung eines lebensbedrohlichen Lungenödems zu erwarten ( $\triangleright$ Abb. 1$)$.

Mostbeck und Partsch untersuchten diese Umverteilung regionaler Blutvolumina (9), indem sie Kompression auf die Beine mit Hilfe aufblasbarer Gummistiefel ausübten und szintigraphisch die Volumenverlagerung erfassten. Unter Kompression war eine Reduktion des Blutvolumens in den Beinen um 33,4\% (25mmHg) bzw. $38,0 \%$ (40 $\mathrm{mmHg}$ ) festzustellen, wohingegen das Blutvolumen der Organe von Thorax, Abdomen und der Leber um 6-7\% zunahm. Lattimer et al. ermittelten mittels Air-Plethysmographie eine Volumenverlagerung von ca. $40 \mathrm{ml}$ pro Bein unter der Einwirkung von MKS der Klassen I und II $(18-32 \mathrm{mmHg})$ an den Unterschenkeln (10). Aufgrund der elastischen Eigenschaften der unteren Hohlvene und der Vielzahl viszeraler Venen gelangt jedoch nur ein Bruchteil des Blutes zum rechten Vorhof. Darüber hinaus wird die Volumenbelastung durch eine Veränderung des Herzzeitvolumens über die Herzfrequenz kompensiert. Aus der Tauchmedizin ist bekannt, dass eine Vorlasterhöhung bei gesunden Herzen auch bei massiver Kompression ohne weiteres toleriert wird. So ist der Organismus bei Immersion in die übliche Sporttauchtiefe von $40 \mathrm{~m}$ einem $100 \mathrm{fach}$ höheren Druck (ca. 3000 mmHg) ausgesetzt, wobei dieser nicht nur auf die Unter-

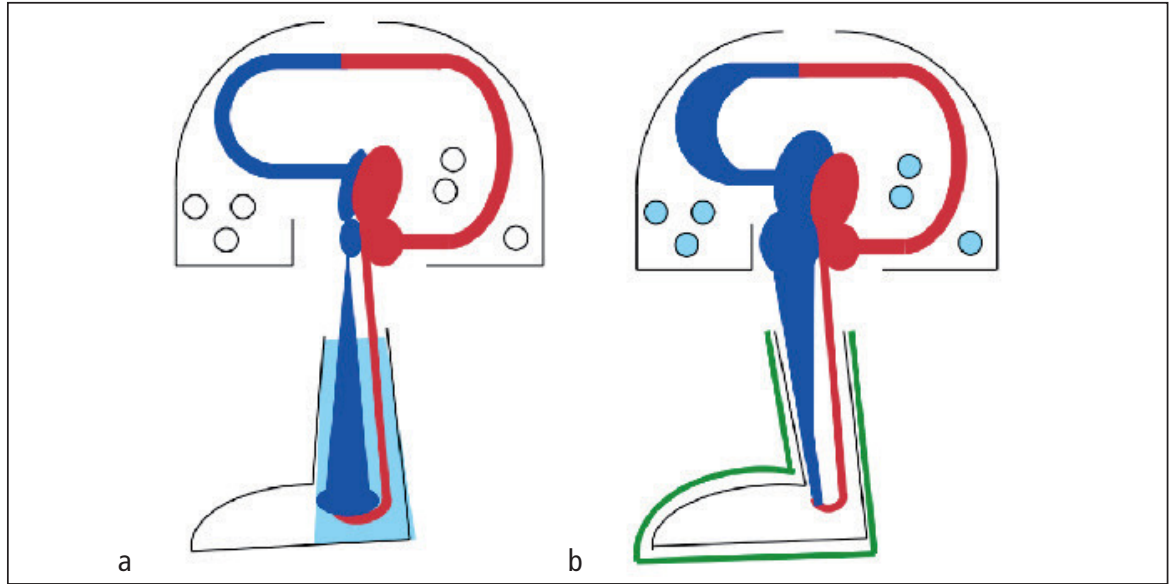

Abb. 1 Der "Kompressionshomunkulus", bestehend aus Bein und Herz mit kleinem Kreislauf, veranschaulicht die Verlagerung des Blutvolumens unter der Wirkung von Kompression. a) Die Venen der Beine (blau) speichern einen erheblichen Anteil des Blutes. Das Beinödem ist hellblau dargestellt. b) Der Kompressionsstrumpf (grün) bewirkt eine Volumenreduktion der ödematösen Extremität. Das Blut der Beinvenen wird dabei über die untere Hohlvene herzwärts verlagert. Dies führt zu einer Volumenbelastung im rechten Vorhof und im rechten Ventrikel. Bei eingeschränkter Herzfunktion kann eine Stauung zur Ausbildung eines Lungenödems (hellblau) führen.

schenkel, sondern auf die gesamte Körperoberfläche (auch den Körperkern) einwirkt.

Im Falle einer strukturellen Herzerkrankung ist jedoch ein verändertes Verhalten des Myokards und der Regulationsvorgänge zu erwarten, welches durch Untersuchungen belegt ist (Dereppe et al. [11], Bain et al. [12]). Ein erhöhtes Volumenangebot im rechten Vorhof bewirkt einen lokalen Druckanstieg sowie getriggert über den resultierenden Wandstress aufgrund der Überdehnung eine vermehrte Expression natriuretischer Peptide (13). Die ESCLeitlinie empfiehlt die Messung von BNP (B-type natriuretic peptide) bzw. dessen Spaltproduktes NT-proBNP als Biomarker für das Vorliegen einer Herzinsuffizienz ( Tab. 2).

Tab. 2

Die Biomarker BNP und NT-proBNP als Marker für die Akuität der Herzinsuffizienz. NT-proBNP weist eine Altersabhängigkeit auf (nach Luchner 2017 [21])
Todd et al. wiesen in einer Gruppe von 102 Probanden mit chronischem Lymphödem an sieben einen erhöhten BNP-Wert nach, bei denen sich auch kardiale Abnormitäten bestätigten (14). Galm et al. konnten in einer vergleichenden Untersuchung an herzgesunden und herzkranken Probanden (NYHA II) mit MKS der Kompressionsklasse II nachweisen, dass ein signifikanter Anstieg des humanen Atrialen Natriuretischen Peptids (hANP) in Folge der MKS nur bei der herzkranken Gruppe festzustellen war, deren Mitglieder aber bereits erhöhte Basalwerte aufwiesen. Der Anstieg des hANP war dabei passager, nur von kurzer Dauer und war nicht begleitet von hämodynamischen Veränderungen (Herzfrequenz, Blutdruckmedian). Die Autoren schlossen daraus, dass Kompressionsthera- 

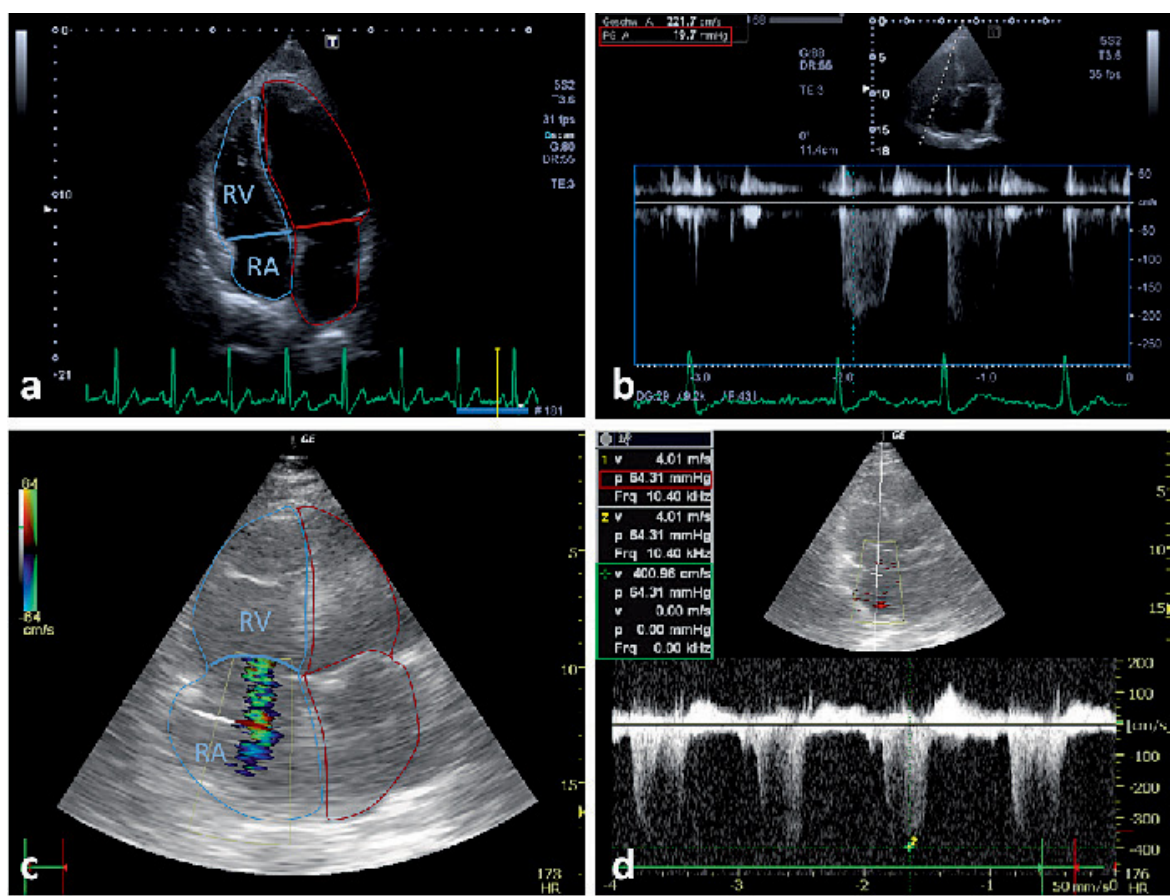

Abb. 2 Herzinsuffizienz in der Echokardiographie. a) Beim Normalbefund sind rechter Ventrikel (RV) und rechter Vorhof (RA) (blau) kleiner als die linkskardialen Kavitäten (rot) und schmiegen sich an diese an. b) Mittels cw-Doppler lässt sich über den Gradienten an der Trikuspidalklappe der Druck im RV ermitteln. Dieser ist mit 19,7mmHg normal. c) Das volumenbelastete Herz zeigt vergrößerte RA und RV (blau). Die rechtskardialen Kavitäten sind sogar größer als die linksseitigen (rot). Die Dilatation des rechten Herzens verursacht zusätzlich eine Insuffizienz der Trikuspidalklappe (grün). d) Der messbare Gradient an der Trikuspidalklappe ist mit $64,31 \mathrm{mmHg}$ pathologisch erhöht. Addiert man einen zentralvenösen Druck von ca. $10 \mathrm{mmHg}$ dazu, ergibt sich ein pulmonal-arterieller Druck von ca. $75 \mathrm{mmHg}$ (Normalwert $\leq 30 \mathrm{mmHg}$ ). Tritt in Folge der Herzinsuffizienz eine herabgesetzte Pumpkraft des linken Herzens hinzu, staut sich das Blut im kleinen Kreislauf - es droht ein Lungenödem. Der Befund korreliert mit einer erheblichen klinischen Beeinträchtigung.

pie mit Unterschenkel-MKS der Kompressionsklasse II bei Patienten mit Herzinsuffizienz im Stadium NYHA II keine Gefährdung darstellt (15). Diese Aussage wird unterstützt von Wilputte et al., die die hämodynamischen Veränderungen nach der Anlage von mehrlagigen Kompressionsbandagen an Patienten einer kardiologischen Intensivstation mit Herzinsuffizienz in den Stadien NYHA III und IV mittels Rechtsherzkatheter (Swan-Ganz-Katheter) untersuchten. Sie konnten feststellen, dass sich die rechtsatrialen und -ventrikulären Druckwerte nach der Anlage der Bandagen zunächst erhöhten, bereits nach 10 Minuten aber wieder zum Ausgangsniveau zurückgekehrt waren, ohne eine nachhaltige klinische Beeinträchtigung zu bewirken. Die Autoren schlussfolgerten aus den Ergebnissen aber, dass die Kompressionsbehandlung bei größeren Ödemmengen we- gen der nicht kalkulierbaren Auswirkungen bei dem genannten Patientengut nicht zur Anwendung kommen sollte (16). Leduc evaluierte mit seiner Arbeitsgruppe die Auswirkung von lokal applizierter Manueller Lymphdrainage $(30-40 \mathrm{mmHg}$ ) an $\mathrm{Pa}$ tienten mit Herzinsuffizienz NYHA III und IV. Sie werteten dazu ebenfalls interventionelle Druckmessungen im kleinen Kreislauf sowie doppler-echokardiographische Messungen und klinisch erfassbare hämodynamische Parameter aus ( Abb. 2). Sie kamen zu dem Ergebnis, dass trotz hochsignifikanter Umfangsreduktion der mit dieser Methode behandelten Extremitäten keine signifikanten Veränderungen hämodynamischer Parameter mit Ausnahme der Herzfrequenz festzustellen waren und formulierten, dass die Entstauung mittels lokal angewendeter Manueller Lymphdrainage (nur Beinbereich) bei Patienten mit
Herzinsuffizienz NYHA III und IV keine Kontraindikation darstellt (17). Gorelik et al. legten 106 Patienten mit dekompensierter Herzinsuffizienz Kompressionsbandagen an, um damit Synkopen durch orthostatisch bedingte Hypotension vorzubeugen. Bei keinem der untersuchten Probanden wurde unter dem gemessenen Blutdruckanstieg eine pulmonale Beeinträchtigung im Sinne eines Lungenödems ausgelöst (18).

Über die Bedeutung einer therapierefraktären bzw. instabilen Hypertonie liegen bislang keine Untersuchungen vor. Es muss jedoch aufgrund der vorliegenden Untersuchungen und übereinstimmenden Beobachtungen bei Volumenverlagerung aufgrund Immersion $(19,20)$ davon ausgegangen werden, dass Kompression in diesen Fällen die Triggerung eines Lungenödems begünstigen kann.

\section{Vorgehensweise in der Praxis}

Die internationale Schweregradeinteilung der Herzinsuffizienz basiert in erster Linie auf anamnestischen Angaben und klinischen Befunden. Differenzialdiagnostisch muss eine kardiale Genese von Ödemen grundsätzlich in Erwägung gezogen werden. Dies gilt in besonderer Weise für bilateral ausgeprägte Ödeme, wohingegen unilaterale Ödeme nicht als Ausdruck einer kardiogenen Systemerkrankung anzusehen sind.

Das Schlüsselsymptom der dekompensierten Herzinsuffizienz (Stadien NYHA III und IV) ist wegen seiner prognostischen Bedeutung die Dyspnoe unter bereits geringer körperlicher Belastung oder Ruhe. Anamnestisch ist $\mathrm{zu}$ erheben, ob Luftnot besteht und unter welchen Bedingungen sie ggf. ausgelöst wird. Luftnot bei flachem Liegen (Orthopnoe) deutet auf eine chronische Lungenstauung als Ausdruck einer schwergradigen bzw. dekompensierten Herzinsuffizienz und spricht ebenso gegen eine unkritische Anwendung von Kompressionstherapie und MLD wie nachweisbare Krepitationen bei der Auskultation der Lunge und eine instabile Hypertonie mit hypertensivem Lungenödem in der Anamnese. 
Sollten Anamnese und klinische Untersuchung keine eindeutige Einordnung der kardiologischen Situation ermöglichen, ist eine weiterführende kardiologische Diagnostik erforderlich (Röntgenuntersuchung des Thorax, NT-proBNP-Messung, Dopplerechokardiographie).

Eine bereits durchgeführte Behandlung kann bei Bestehen einer stabilen Herzinsuffizienz fortgesetzt werden, soweit darunter keine Dyspnoe bzw. Herzbeschwerden beklagt werden.

Bei relevanter kardialer Komorbidität ist ggf. einem stationären Setting der Vorzug zu geben.

\section{Schlussfolgerung}

Die zum aktuellen Zeitpunkt publizierten Fallbeschreibungen und die Ergebnisse der bislang wenigen vorliegenden experimenteller Studien lassen folgende Schlussfolgerungen zu:

1. Die Herzinsuffizienz stellt keine Gegenanzeige per se für Kompressionstherapie und Manuelle Lymphdrainage dar.

2. In den Krankheitsstadien NYHA I und NYHA II ist eine Behandlung mittels Kompressionsverbänden und Kompressionsstrümpfen sowie MLD weitgehend ohne Einschränkung möglich.

3. In den Stadien NYHA III und IV ist eine vorsichtige Entstauungstherapie bei strenger Indikationsstellung unter klinischer und hämodynamischer Kontrolle eingeschränkt möglich.

\section{Interessenkonflikt}

Nach Angaben des Autors bestehen keine Interessenkonflikte.

\section{Ethische Richtlinien}

Für das Manuskript wurden keine Studien an Menschen oder Tieren durchgeführt.

\section{Literatur}

1. Wienert V, Gerlach H, Gallenkemper G et al. Leitlinie Medizinischer Kompressionsstrumpf (MKS) Phlebologie 2006; 35: 315-320.

2. http://www.awmf.org/uploads/tx_szleitli nien/037-005_S2_Phlebologischer_Kompressionsverband_PKV_2009_abgelaufen.pdf (Download: 12/16/2017)

3. http://www.awmf.org/uploads/tx_szleitli nien/058-0011_S2k_Diagnostik_und_Therapie_der_Lymphoedeme_2017-05.pdf (Download: $12 / 16 / 2017$ )

4. Vaassen MM. Manual Lymph Drainage in a Patient with Congestive Heart Failure: A Case Study. Ostomy Wound Manage 2015; 61(10): 38-45.

5. McCardell CS, Berge KH, Ijaz M et al. Acute pulmonary edema associated with placement of waist-high, custom-fit compression stockings. Mayo Clin Proc 1999; 74(5): 478-480.

6. Andriessen A, Apelqvist J, Mosti G et al. Compression therapy for venous leg ulcers: risk factors for adverse events and complications, contraindications - a review of present guidelines. J Eur Acad Dermatol Venereol 2017; 31(9): 1562-1568. doi: 10.1111/jdv.14390. Epub 2017 Jul 31.

7. Ponikowski P, Voors AA, Anker SD et al. 2016 ESC Guidelines for the diagnosis and treatment of acute and chronic heart failure. Eur Heart J (2016) 37;2129-2200. doi: 10.1093/eurheartj/ehw128

8. Dolgin M. The Criteria Committee of the New York Heart Association. Nomenclature and Criteria for Diagnosis of Diseases of the Heart and Great Vessels. 9th ed. Boston, Mass: Little, Brown \& Co 1994; 253-256.

9. Mostbeck A, Partsch H. Umverteilung regionaler Blutvolumina durch Dihydroergotamin und Beinkompression. Med Klin 1978; 73: 801-806.

10. Lattimer CR, Kalodiki E, Azzam M, Geroulakos G. Haemodynamic Performance of Low Strength Below Knee Graduated Elastic Compression Stockings in Health, Venous Disease, and Lymphoedema. Eur J Vasc Endovasc Surg 2016; 52 . 105-112.
11. Dereppe H, Hoylaerts M, Renard M et al. [Hemodynamic impact of pressotherapy]. J Mal Vasc (1990);15(3):267-9 (Abstract only, FRENCH)

12. Bain RJ, Tan LB, Murray RG et al. Central haemodynamic changes during lower body positive pressure in patients with congestive cardiac failure. Cardiovasc Res. 1989; 23(10): 833-837.

13. Wilkins MR, Gammage MD, Lewis HM et al. The effect of lower-body positivepressure on blood pressure, plasma ANP concentration and sodium and waterexcretion in healthy volunteers and cardiac transplant recipients. Cardiovasc Res 1988; 22: 231-235

14. Todd J, Austwick T, Berridge D et al. B-type natriuretic peptide in lymphedema. Lymphology 2011; 44(1): 29-34.

15. Galm O, Jansen-Genzel, von Helden J, Wienert V. Plasma human atrial natriuretic peptide under compression therapy in patients with chronic venous insufficiency with or without cardiac insufficiency. Vasa 1996; 25(1): 48-53.

16. Wilputte F, Renard M, Venner JP. Hemodynamic Response to Multilayered Bandages Dressed on a Lower Limb of Patients with Heart Failure. Eur J Lym 2005; Vol XV(45): 1-4.

17. Leduc O, Crasset V, Leleu C et al. Impact of Manual Lymphatic Drainage on Hemodynamic Parameters in Patients with Heart Failure and Lower Limb Edema. Lymphology 2011; 44: 13-20.

18. Gorelik O, Almoznino-Sarafian D, Litvinov V et al. Seating-induced postural hypotension is common in older patients with decompensated heart failure and may be prevented by lower limb compression bandaging. Gerontology. 2009;55(2):138-44. doi: 10.1159/000141920. Epub 2008 Jun 27.

19. Peacher DF, Martina SD, Otteni CE et al. Immersion pulmonary edema and comorbidities: case series and updated review. Med Sci Sports Exerc 2015; 47(6): 1128-1134. doi: 10.1249/MSS.0000000000000524.

20. Gempp E, Demaistre S, Louge P. Hypertension is predictive of recurrent immersion pulmonary edema in scuba divers. Int J Cardiol. 2014 Mar 15;172(2):528-9. doi: 10.1016/j.ijcard.2014.01.021. Epub 2014 Jan 22.

21. Luchner A, von Haehling S, Holubarsch $C$ et al. [Indications and Clinical Implications of the Use of the Cardiac Markers BNP and NT-proBNP]. [Article in German]. Dtsch Med Wochenschr 2017; 142(5): 346-355. 